INPLASY

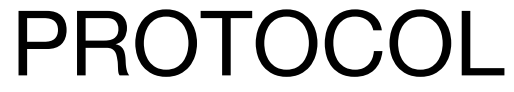

To cite: Zou et al. A metaanalysis of the incidence of sarcopenia in diabetic patients in China. Inplasy protocol 2020110023. doi:

10.37766/inplasy2020.11.0023

Received: 06 November 2020

Published: 06 November 2020

Corresponding author:

Linlin Zhang

527122729@qq.com

Author Affiliation:

Harbin University

Support: National Natural

Science Found.

Review Stage at time of this submission: Data analysis.

Conflicts of interest:

No conflict of interest.

\section{A meta-analysis of the incidence of sarcopenia in diabetic patients in China}

Review question / Objective: Observe the incidence of sarcopenia in Chinese diabetic patients.

Condition being studied: Sarcopenia in diabetic patients in China.

Information sources: Computer search of CNKI, Weipu, Wanfang, CBM, Pubmed, Web Of Science, Embase database, the search time limit is the establishment of each database until October 24, 2020. Search for relevant documents published in these databases. The English search term is: Diabetes Mellitus/Sarcopenia/China; the Chinese search term is: 2性糖尿病/糖尿病/肌少症/肌肉减少症/骨骼肌减少症, using a combination of subject terms + free words to search.

INPLASY registration number: This protocol was registered with the International Platform of Registered Systematic Review and Meta-Analysis Protocols (INPLASY) on 06 November 2020 and was last updated on 06 November 2020 (registration number INPLASY2020110023).

\section{INTRODUCTION}

Review question / Objective: Observe the incidence of sarcopenia in Chinese diabetic patients.

Condition being studied: Sarcopenia in diabetic patients in China.

\section{METHODS}

Search strategy: The English search term is: Diabetes Mellitus/Sarcopenia/China; the Chinese search term is: 2性糖尿病/糖尿病/肌 少症/肌肉减少症/骨骼肌减少症, using a combination of subject terms + free words to search. 
Participant or population: Chinese diabetic patients aged 60 or above.

Intervention: Observational research without intervention.

Comparator: Chinese healthy persons aged 60 or above.

Study designs to be included: Crosssectional study and case-control study.

Eligibility criteria: 1. Literature inclusion criteria: Study type: Observational study that can extract the number of cases of sarcopenia Research population: Diagnosis of diabetes and sarcopenia is clear (the diagnosis of sarcopenia includes muscle mass testing, grip strength testing, and $6 \mathrm{~m}$ pace testing); age $\geq 60$ years old; no serious diabetes complications 2. Exclusion criteria: Duplicate literature; reviews, conferences, animal experiments; no clear report on the number of sarcopenia cases; inconsistent research content; severe diabetic complications.

Information sources: Computer search of CNKI, Weipu, Wanfang, CBM, Pubmed, Web Of Science, Embase database, the search time limit is the establishment of each database until October 24, 2020. Search for relevant documents published in these databases.

Main outcome(s): A total of 338 relevant documents were retrieved, and 12 were included in the meta-analysis after preliminary screening and re-screening, including 10 cross-sectional studies, 2 case-controls, and 2916 diabetic patients.

Quality assessment / Risk of bias analysis: Two researchers who have been trained in literature quality evaluation will carry out literature screening, quality evaluation and data extraction based on the literature inclusion and exclusion criteria (if any differences arise, they will be resolved through rechecking or discussion or consultation with relevant experts). The case-control study was scored according to the Newcastle-Qttawa Scale (NOS) (total score of 9 points). The evaluation content included the selection of the study population (a total of four items, a full score of 4), and the comparability between groups ( One item, full score of 2), measurement of results/exposure factors (a total of three items, full score of 3); the cross-sectional study is based on the quality evaluation recommended by the Agency For Healthcare Research and Quality (AHRQ) The standard is scored (a total of 11 points). The evaluation standard includes eleven items, each with "yes", "no" and "unclear" as answers, 1 point for "yes" and "no" or "Unclear" counts as 0 points. The higher the score, the better the quality of the literature.

Strategy of data synthesis: Using Stata16 and ReviewMan5.3 for statistical analysis, taking the incidence $p$ and its standard error se as the effect size, the calculation formula for $P$ is as follows: $p=x / n$, $\operatorname{se}(p)=\sqrt{p}(1-p) / n$ (x Is the number of events, $n$ is the total number of observation objects) When the occurrence rate $P$ satisfies the normal distribution, this formula can be used. Meta results are heterogeneous with 12 greater than $50 \%$. When the heterogeneity is strong, random effects model analysis is used, Egger linear regression and Begg rank correlation method are used to detect publication bias, and sensitivity analysis detects the stability of Meta results. All statistical results $\mathrm{P}<0.05$ are considered statistically significant.

Subgroup analysis: When the heterogeneity is large, perform a subgroup analysis to explore the source of the heterogeneity. The subgroup analysis includes: sample size, patient source, and muscle mass measurement method.

Sensibility analysis: Sensitivity analysis of the literature included in the meta-analysis. If the results of each study are within the overall confidence interval, the metaanalysis results are stable

Country(ies) involved: China.

Keywords: China, diabetes, sarcopenia, incidence, meta. 
Contributions of each author/ email:

Author 1 - Hao Zou. 1790399324@qq.com

Author 2 - Gaoyue Ren.

Author 3 - Siqin Liao.

Author 4 - Yang Liu.

Author 5 - Linlin Zhang.527122729@qq.com 